\title{
Post hoc analysis of a randomized controlled trial comparing concurrent chemoradiation with cisplatin versus nimotuzumab-cisplatin, focusing on acute oral mucositis
}

\author{
Vanita Noronha ${ }^{1 \dagger}$, Vijay M. Patil ${ }^{1 \dagger}$, Gunjesh Kumar Singh ${ }^{1 \dagger}$, Amit Joshi ${ }^{1}$, Nandini Menon ${ }^{1}$, Sarbani Ghosh Lashkar ${ }^{2}$, \\ Vijayalakshmi Mathrudev', Kavita Nawale Satam and Kumar Prabhash ${ }^{1 *}$
}

\begin{abstract}
Background: Acute oral mucositis has been infrequently studied in the patients with head and neck squamous cell carcinoma (HNSCC) receiving once-weekly cisplatin-based chemoradiotherapy (CRT). Hence, this analysis was conducted to explore the various aspects of the same.

Results: The overall incidence of mucositis was $96.9 \%(n=508)$ and of grade $3-5$ mucositis was $61.3 \%(n=321)$. The overall incidence of oral mucositis was similar in both the arms (CCRT and NCRT) ( $p$ value $=0.58$ ) while grade $3-5$ mucositis was more common in the NCRT arm ( $p$ value $=0.01$ ). Out of all factors listed, the presence of nimotuzumab was the only significant risk factor for the development of grade 3 or more oral mucositis ( $p$ value $=$ $0.01)$; ( $\mathrm{OR}=1.64,95 \% \mathrm{Cl} 1.15-2.32)$. Delays in the treatment delivery were similar in both the arms.
\end{abstract}

Conclusion: Acute oral mucositis is a common occurrence in locally advanced-HNSCC patients receiving chemoradiotherapy. Nimotuzumab is a significant factor for development of grade 3 and above oral mucositis.

Keywords: Acute oral mucositis, Head and neck cancer, Chemoradiation

\section{Background}

Cisplatin-based chemoradiotherapy (CRT) has been the standard treatment for patients with locally advanced head and neck squamous cell carcinomas (LA-HNSCC), given with the aim of preserving function and improving survival [1]. However, this intensive chemoradiotherapy is associated with significant acute and late toxicities [2]. The data with regard to the acute adverse events especially oral mucositis with $100 \mathrm{mg} / \mathrm{m}^{2}$ of 3-weekly CRT is well documented; however, there is a dearth of data in

\footnotetext{
* Correspondence: kumarprabhashtmh@gmail.com

${ }^{\dagger}$ Vanita Noronha, Vijay M Patil, and Gunjesh Kumar Singh are co-first authors. 'Department of Medical Oncology, Tata Memorial Hospital, Mumbai 400012, India

Full list of author information is available at the end of the article
}

case of weekly CRT regime. While comparing 3-weekly and once weekly CRT regimes in a retrospective study, Tsan et al. found a higher rate of oral mucositis and overall toxicity in the latter [3]. In a meta-analysis by Szturz et al, higher dose cisplatin given three to four weekly was found less toxic when compared to low dose cisplatin $\left(<50 \mathrm{mg} / \mathrm{m}^{2}\right)$ given once weekly [4]. Oral mucositis can also complicate oral candidiasis which is commonly observed in these patients and increase susceptibility to fungemia [5]. High-grade oral mucositis and their complications often lead to treatment delay resulting in poor survival outcomes.

In our institution, weekly CRT with cisplatin at a dose of $30 \mathrm{mg} / \mathrm{m}^{2}$ is being used for chemoradiotherapy for 14 years 
(2006). Theoretically, once weekly CRT appears better tolerable but there is literary evidence of it being rather more toxic. There is no information till date regarding the pattern of oral mucositis, its presentation, peak, and recovery in patients who are given once weekly CRT. Patients with dysphagia due to tumor proximity and those with their swallowing apparatus in the radiotherapy treatment field are at high risk for development of toxicities. Hence, it becomes exceedingly important to keep these patients (HNSCC on chemoradiotherapy) well informed about the time and extent of occurrence of mucositis and the related complications possible during the treatment.

The purpose of this study was to evaluate the peak incidence, severity, and the pattern of presentation of oral mucositis along with the factors affecting it in a cohort of patients with HNSCC undergoing concurrent chemoradiotherapy. The knowledge will help in patient education on the treatment and its effects and will also guide clinical support services.

\section{Methods}

\section{Study details}

This was a retrospective post hoc analysis of a prospective randomized controlled trial comparing weekly concurrent cisplatin $\left(30 \mathrm{mg} / \mathrm{m}^{2}\right)$ (CCRT) with weekly NimotuzumabCRT (NCRT) from 2012 to 2018 [6]. Approval for this study was obtained from the Institutional Review Board. A written informed consent was obtained from every patient before participation. Newly diagnosed treatment-naive adult patients with non-metastatic, stage III or IV LAHNSCC arising in the oropharynx, larynx, hypopharynx, or oral cavity were eligible for the study. The other criteria were a Karnofsky performance status $\geq 70$ and adequate hematological, renal, and hepatic functions. Patients with tumors originating in the nasopharynx, salivary gland, or nasal cavity and those who had received immunotherapy or prior radiotherapy to the head and neck region were excluded. In both the arms, cisplatin was dosed at $30 \mathrm{mg} / \mathrm{m}^{2}$ weekly along with radiation therapy and supportive medications. Nimotuzumab was administered weekly in the NCRT arm intravenously as a 200-mg flat dose in $250 \mathrm{~mL}$ normal saline over $60 \mathrm{~min}$ without any premedication. Patients received prior hydration, 5HT3 antagonists along with dexamethasone and/or neurokinin-1 receptor antagonist as antiemetic prophylaxis. All the patients were also provided weekly oral care by the dentists in addition to proper education on skin care by pharmacists and nurses. The toxicities were graded using the Common Terminology Criteria for Adverse Events (NCI-CTCAE) Version 4.03 [7].

\section{Data collection}

Data related to oral mucositis during the course of chemoradiotherapy, i.e., from week 0 to 7 of treatment was collected. The information on the occurrence of other acute toxicities was also captured and collected along with chemotherapy (CCRT and NCRT) delivery details. The entire data was entered in an excel sheet and the following details were noted:

1. Incidence, severity of oral mucositis in both CCRT and NCRT arm.

2. Factors affecting acute oral mucositis-age (older, i.e., $\geq 60$ years vs younger), gender, Eastern Cooperative Oncology Group performance score (ECOG-PS), stage of primary, site of primary, the technique of radiotherapy used, and chemotherapy regimen (weekly CCRT and NCRT).

3. The severity of other acute adverse events such as rise in serum creatinine level, odynophagia, dysphagia, weight loss, and dermatitis were also noted. Purpose of this detailed data collection was to see their association with oral mucositis.

4. The impact of oral mucositis on treatment delay and hospitalization rates due to severe oral mucositis was also studied.

\section{Statistical analysis}

The incidence rates of acute mucositis were computed and the factors affecting it were sought, following which, the cumulative incidence rate was calculated using a competing risk analysis. SPSS version 20 was used for statistical analysis. Grays test was used to compare the incidence rates between nimotuzumab and nonnimotuzumab regimens. To see the relationship between mucositis and various factors, Fisher test (2-sided) and binary logistic regression analysis were used for univariate analysis. The relationship between complications and mucositis was studied by using fisher test. $p$ value $\leq 0.05$ was considered significant.

\section{Results \\ Incidence of mucositis}

Out of 536 patients, acute adverse events were captured in 524 patients. The overall incidence of mucositis was 96.9\% $(n=508)$ while for grade $3-5$ mucositis it was $61.3 \%(n=321)$. Out of these 524 patients, 260 were in the CCRT group and 264 in the NCRT group. The overall incidence of oral mucositis was $96.9 \%(n=252)$ while it was $55.8 \%(n=145)$ for grade $3-5$ in the CCRT group. In comparison, in the NCRT group, the overall incidence was $97 \%(n=256)(p$ value $=0.58)$ while that of grade $3-5$ mucositis was $66.7 \%(n=176)(p$ value $=$ 0.01 ).

The cumulative incidence of mucositis in both the arms is shown in Fig. 1. The temporal evolution of the pattern of mucositis per week of all patients and patients in each arm according to the treatment regimen is 


\section{Cumulative incidence curve for mucoitis}

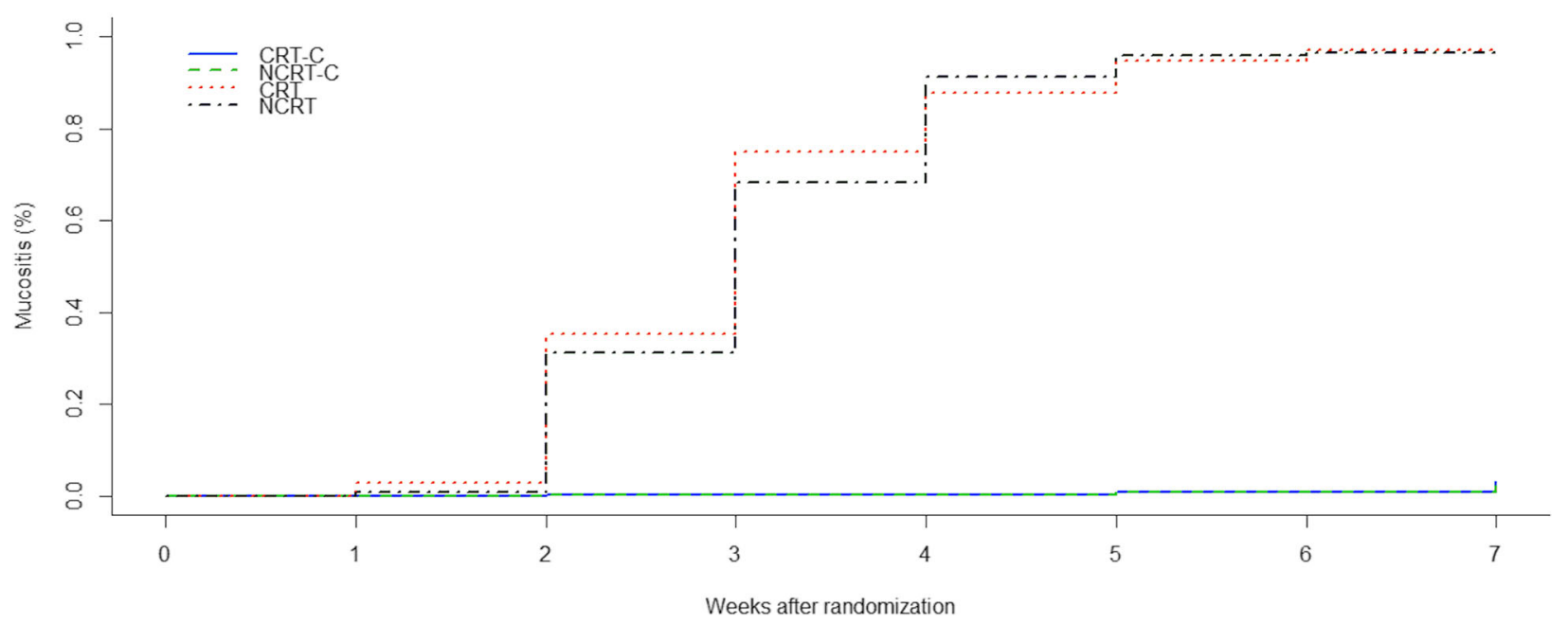

Fig. 1 Cumulative incidence of mucositis

shown in Figs. 2, 3, and 4. The median time to develop any grade mucositis was 3 weeks $(95 \%$ CI $2.9-3.1)$ while it was 7 weeks (95\%CI 6.7-7.2) for grade 3-5 mucositis.

\section{Factors associated with mucositis}

We found that none of the listed factors was associated with an increased risk of development of any grade mucositis (Table 1). However, the presence of nimotuzumab in the NCRT regime did add to the risk of grade 3 or more mucositis $(p$ value $=0.01)$; $(\mathrm{OR} 1.64,95 \% \mathrm{CI}$ 1.15-2.32) (Table 2).

\section{Impact of mucositis on treatment delivery}

The presence of oral mucositis delayed chemotherapy for 3 or more days in $32.5 \%(n=87)$ and $30.6 \%(n=82)$ patients in the CCRT and NCRT arms respectively ( $p$ value $=0.642)$. Again, patients who required chemotherapy dose modification due to mucositis were $7.8 \%(n=21)$ and $9.7 \%(n=26)$ in the CCRT and NCRT arm respectively ( $p$ value $=0.445)$. Patients who received cumulative chemotherapy with $<7$ cycles of weekly cisplatin due to mucositis were $12.7 \%(n=34)$ and $13.1 \%(n=35)$ in the CCRT and NCRT arm respectively $(p$ value $=0.445)$. Patients who had a delay of radiotherapy for 3 or more days were $3.7 \%(n=10)$ and $4.5 \%(n=12)$ in CCRT and NCRT arm $(p$ value $=0.642)$, respectively.

\section{Association of oral mucositis with other toxicities}

We observed that other toxicities such as dysphagia, weight loss, and dermatitis of any grade were also

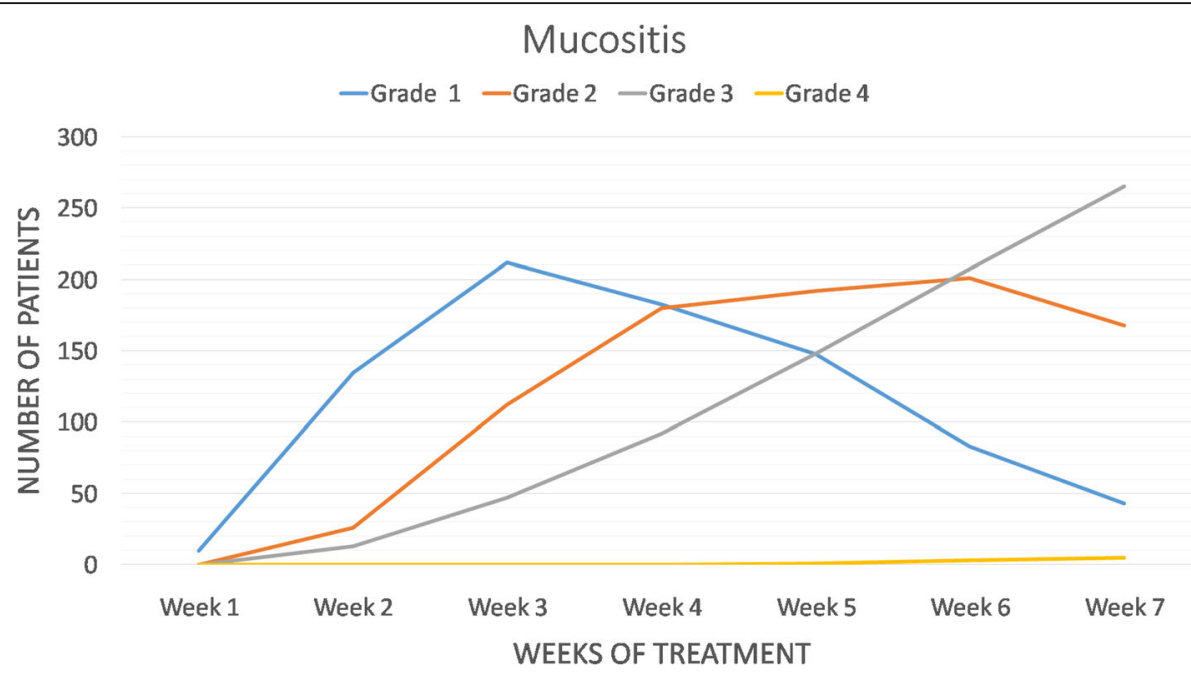

Fig 2 Temporal evolution of the pattern of mucositis per week 


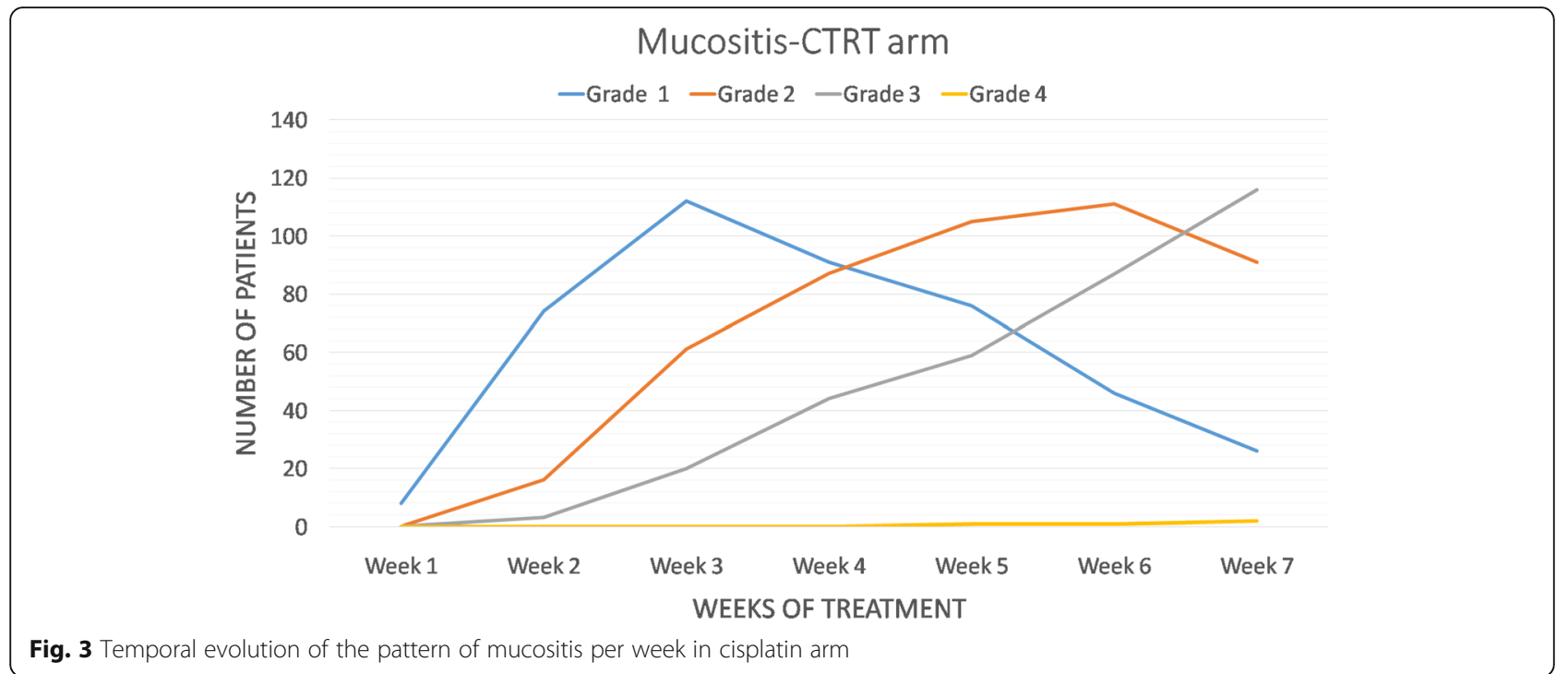

associated with oral mucositis and this association was mostly seen with higher grades (grade 3 and above) of the former (Table 3). Out of all patients with acute adverse events, the ones with any grade of oral mucositis and required admission and indoor care anytime during the course of treatment were 114 (21.8\%). 82 (25.5\%) out of all patients with grade 3 or above oral mucositis $(n=$ 321) needed admission for supportive care. Further, the hospitalization rates were also not different between patients with any grade and grade 3 or above oral mucositis $(p$ value $=0.541)$.

\section{Discussion}

Different studies have been conducted to evaluate the frequency and possible risk factors of concurrent chemoradiotherapy (CTRT) induced oral mucositis. However, the details of the mechanism of oral mucositis development are completely unknown, and its control during CTRT remains challenging [8]. To the best of our knowledge, this is the first longitudinal study to investigate the association between oral mucositis and different risk factors during CTRT. The overall incidence of mucositis in our patients was $96.9 \%(508 / 524)$ and grade 3 and above were seen in $61.3 \%(321 / 524)$ patients. The overall incidence of oral mucositis was similar in both the arms ( $p$ value $=0.58)$; however, that of grade 3-5 was more in the NCRT arm $(p$ value $=0.01)$. We also found that the rate of hospitalization increased with increasing severity of mucositis and $25.5 \%$ (82) patients with grade 3 or more mucositis required indoor care.

In the landmark studies by Bernier et al. and Cooper et al., they noted that cumulative grade 3 or above

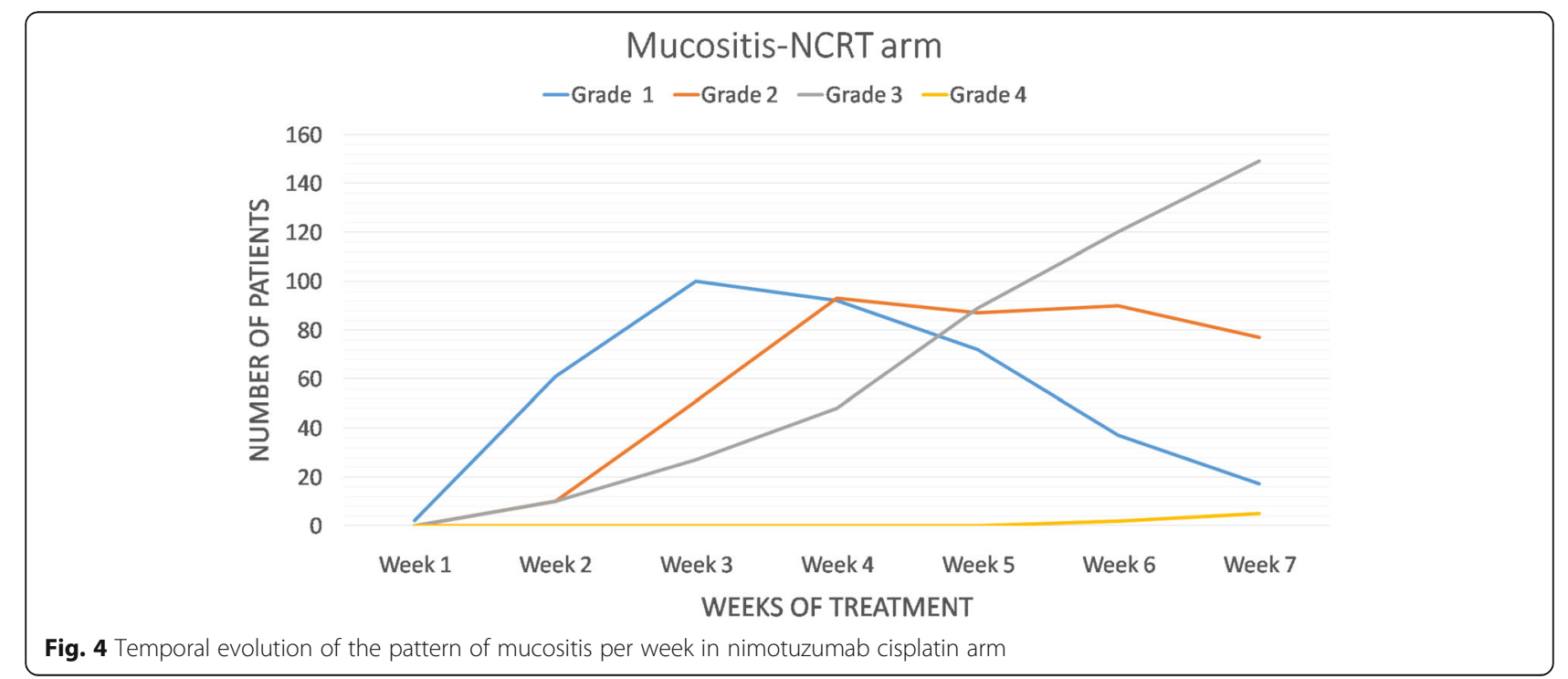


Table 1 Factors affecting any grade oral mucositis

\begin{tabular}{|c|c|c|c|}
\hline & Number of patients $(n)$ & Any grade mucositis ( $n, \%)$ & Univariate $p$ value \\
\hline \multicolumn{4}{|l|}{ Arm } \\
\hline Cisplatin & 260 & $252(96.9 \%)$ & 1.000 \\
\hline Cisplatin-nimotuzumab & 264 & $256(96.9 \%)$ & \\
\hline \multicolumn{4}{|l|}{ Gender } \\
\hline Male & 445 & $431(96.9 \%)$ & 1 \\
\hline Female & 79 & 77 (97.5\%) & \\
\hline \multicolumn{4}{|l|}{ ECOG PS } \\
\hline PS 0 & 116 & $115(99.1 \%)$ & 0.216 \\
\hline PS 1-2 & 408 & $393(96.3 \%)$ & \\
\hline \multicolumn{4}{|l|}{ Stage } \\
\hline III & 163 & $158(96.9 \%)$ & 1 \\
\hline IV & 361 & $350(96.9 \%)$ & \\
\hline \multicolumn{4}{|l|}{ Radiotherapy technique } \\
\hline Conventional & 458 & $443(96.7 \%)$ & 0.706 \\
\hline IMRT & 66 & 65 (98.5\%) & \\
\hline \multicolumn{4}{|l|}{ Age (continuous variable) } \\
\hline Age & 536 & - & - \\
\hline
\end{tabular}

mucosal adverse events were more in the CCRT arm (cisplatin $100 \mathrm{mg} / \mathrm{m}^{2}, 3$-weekly) in comparison to the radiation alone arm that is $41 \%$ vs. $21 \% ; p=0.001$ and $44.5 \%$ vs. $21.3 \%, p<0.001$, respectively [9, 10]. Many studies like these in the literature have described and reported the occurrence of acute oral mucositis with 3- weekly cisplatin, but there only a handful of them studying the same with the weekly cisplatin regime. Tsan et al. compared weekly and 3-weekly cisplatin regimes in a small cohort of 55 patients and found that $22(91.7 \%)$ patients in the former group $(n=24)$ had grade 3 and above oral mucositis [3]. Noronha et al., in a randomized

Table 2 Factors affecting grade 3-4 oral mucositis

\begin{tabular}{|c|c|c|c|}
\hline & Number of patients $(n)$ & Grade 3-4 mucositis ( $n, \%)$ & Univariate $p$ value \\
\hline \multicolumn{4}{|l|}{ Arm } \\
\hline Cisplatin & 260 & $145(55.8 \%)$ & 0.012 \\
\hline Cisplatin-nimotuzumab & 264 & $176(66.7 \%)$ & \\
\hline \multicolumn{4}{|l|}{ Gender } \\
\hline Male & 445 & $275(61.8 \%)$ & 0.616 \\
\hline Female & 79 & $46(58.2 \%)$ & \\
\hline \multicolumn{4}{|l|}{ ECOG PS } \\
\hline PS 0 & 116 & $68(58.6 \%)$ & 0.519 \\
\hline PS 1-2 & 408 & $253(62 \%)$ & \\
\hline \multicolumn{4}{|l|}{ Stage } \\
\hline III & 163 & $98(60.1 \%)$ & 0.771 \\
\hline IV & 361 & $223(61.8 \%)$ & \\
\hline \multicolumn{4}{|l|}{ Radiotherapy technique } \\
\hline Conventional & 458 & $279(60.9 \%)$ & 0.787 \\
\hline IMRT & 66 & $42(63.6 \%)$ & \\
\hline \multicolumn{4}{|l|}{ Age (continuous variable) } \\
\hline Age & 536 & - & - \\
\hline
\end{tabular}


Table 3 Association of acute oral mucositis with other toxicities

\begin{tabular}{lllll}
\hline Adverse event & $\begin{array}{l}\text { Grade of } \\
\text { adverse } \\
\text { event }\end{array}$ & \begin{tabular}{l} 
Mucositis \\
\cline { 3 - 4 }
\end{tabular} & $\begin{array}{l}\text { Grade } \mathbf{0 - 2} \\
(\boldsymbol{n}=\mathbf{2 0 3})\end{array}$ & $\begin{array}{l}\text { Grade3-5 } \\
(\boldsymbol{n}=\mathbf{3 2 1})\end{array}$ \\
\hline Rise in serum creatinine & Any grade & $17(8.4 \%)$ & $32(10 \%)$ & 0.645 \\
& Grade 3-5 & - & $2(0.6 \%)$ & 0.524 \\
Odynophagia & Any grade & $192(94.6 \%)$ & $317(98.8 \%)$ & 0.007 \\
Dysphagia & Grade 3-5 & $38(18.7 \%)$ & $169(52.6 \%)$ & $<0.0001$ \\
& Any grade & $160(78.8 \%)$ & $295(91.9 \%)$ & $<0.0001$ \\
Weight loss & Grade 3-5 & $33(16.3 \%)$ & $122(38.0 \%)$ & $<0.0001$ \\
& Any grade & $50(24.6 \%)$ & $243(75.7 \%)$ & $<0.0001$ \\
Dermatitis & Grade 3-5 & $0(0)$ & $5(1.6 \%)$ & 0.162 \\
& Any grade & $168(82.8 \%)$ & $304(94.7 \%)$ & $<0.0001$ \\
& Grade 3-5 & $38(18.7 \%)$ & $111(34.6 \%)$ & $<0.0001$ \\
\hline
\end{tabular}

phase III controlled trial from India, found a similar rate of severe acute oral mucositis (grade 3 or above) in once weekly and once 3 -weekly cisplatin regimens $(17.3 \%$ vs $18.1 \%, p$ value $=0.9)[11]$. Further, a systematic review of literature and meta-analysis by Szturz et al. showed that patients in the weekly cisplatin arm experienced a higher rate of grade 3 or above acute oral mucositis (75\% vs $40 \%, p=.0202$ ) [4]. Similarly, the prevalence and severity of acute oral mucositis in our study were high (61.3\%).

Radiotherapy induced mucositis begins in the 2nd or 3rd week of treatment and reaches its peak at around 5th to 6th week [12]. Similarly, in our study the median time to oral mucositis was 3 weeks and it peaked at 7th week.

Along with the type of chemoradiotherapy, a variety of factors including age, nutritional status, type of malignancy, pretreatment oral condition, oral care during treatment, and pretreatment neutrophil counts are proposed to be associated with the development of acute oral mucositis in patients with HNSCC [13]. In an Indian study by Suresh et al. age $>40$ years, ECOG PS $>2$, total leucocyte count $<3000 / \mu \mathrm{L}$, elevated erythrocyte sedimentation rate, serum albumin $<3 \mathrm{gm} / \mathrm{dL}$, a primary tumor of stage 3 or more, comorbid conditions, nutritional status, oral hygiene, and tobacco use were taken as the risk factors. They used these risk factors to calculate a score. The higher the score, the more is the chance of developing acute oral mucositis [14]. Out of all risk factors listed in our study, we found that the addition of nimotuzumab was significantly linked with the development of grade 3 and above acute oral mucositis $(p$ value $=0.01)$.

In a systematic review of literature, which included 33 studies to see the incidence, severity, and outcomes of oral mucositis, oral pain, weight loss, dysphagia, dehydration, and use of analgesics/opioid were reported as the important symptoms of oral mucositis [15]. Out of these, weight loss was the most common symptom seen in 10/33 studies. Notably, the rates of hospitalization due to acute oral mucositis were higher in patients in the chemoradiotherapy group than those who received radiotherapy alone (6\% vs. $2 \%$ group) [16]. One needs to pay attention that severe oral mucositis is significantly associated with more total parenteral nutrition support and parenteral narcotic therapy. Also, there is a higher chance of infection which may further increase the duration of hospital stay and cost of inpatient supportive care [17]. In our patients, $21.8 \%$ and $25.5 \%$ patients with any grade and grade 3 and above oral mucositis required inpatient care, which was high but the actual number of patients who were admitted because of oral mucositis per se was not captured and this remains one of the limitations of our study.

It should be noted that oral mucositis can lead to treatment delay and treatment (chemotherapy and radiotherapy dose) modification. However, in both the CCRT and NCRT arms of our study, no difference in delay of chemotherapy and radiotherapy delivery was seen and cumulative doses of chemotherapy and radiotherapy were similar as well. In various studies, planned or unplanned treatment modifications have been reported frequently, but the extent or link to mucositis is rarely noted by the authors [15].

To our knowledge, this study represents the largest and most comprehensive report of acute oral mucositis and its outcomes in patients with LA-HNSCC treated with radiotherapy and concurrent chemotherapy. Overall, the current data indicates that patients continue to require supportive care for a range of treatment-related toxicities, including acute oral mucositis, particularly beginning in the first 3-4 weeks, until the final weeks of treatment with symptoms improving thereafter.

\section{Conclusion}

This study confirms that patients with LA-HNSCC undergoing concurrent chemotherapy with radiotherapy experience a range of treatment-related toxicities including oral mucositis. Also, nimotuzumab is a significant factor associated with development of grade 3 and above oral mucositis. Hence, there is a need for contemporary evidence base to help guide the delivery of timely clinical and supportive care to these patients during treatment.

\section{Abbreviations}

HNSCC: Head and neck squamous cell carcinoma; CRT: Cisplatin-based chemoradiotherapy; NCRT: Weekly nimotuzumab-cisplatin-based chemoradiotherapy; LA-HNSCC: Locally advanced head and neck squamous cell carcinomas; CCRT: Weekly concurrent cisplatin $\left(30 \mathrm{mg} / \mathrm{m}^{2}\right)$; NCl-

CTCAE: Common Terminology Criteria for Adverse Events; ECOG-PS : Eastern Cooperative Oncology Group performance score; CTRT: Concurrent chemoradiotherapy

Acknowledgements

None 


\section{Authors' contributions}

All authors contributed to the study conception and design. Material preparation, data collection, and analysis were performed by all. The first draft of the manuscript was written by KP, VN, and VMP, and all authors commented on previous versions of the manuscript. All authors read and approved the final manuscript.

\section{Funding}

None

\section{Availability of data and materials}

The authors confirm that the data supporting the findings of this study are available within the article.

\section{Declarations}

\section{Ethics approval and consent to participate}

The study protocol was approved by the Institutional Ethics Committee, Advanced Centre for Treatment, Research and Education in Cancer (ACTREC), Mumbai, 410210, India and was registered with the Clinical Trial Registry of India (trial registration identifier CTRI/2014/09/004980). It was conducted according to the Declaration of Helsinki and good clinical practice guidelines, and it was monitored by the TMC Data Safety and Monitoring Committee. A written informed consent was obtained from every patient before participation.

\section{Consent for publication}

Not applicable

\section{Competing interests}

The authors declare that they have no competing interests.

\section{Author details}

'Department of Medical Oncology, Tata Memorial Hospital, Mumbai 400012, India. ${ }^{2}$ Department of Radiotherapy, Tata Memorial Hospital, Mumbai 400012, India.

Received: 21 January 2021 Accepted: 7 May 2021

Published online: 22 May 2021

\section{References}

1. Singh GK, Noronha V, Patil VM, Menon N, Joshi A, Prabhash K. Safety and efficacy of concurrent chemoradiotherapy for head-and-neck cancers in older versus younger patients: post hoc analysis of a randomized controlled trial. Cancer Res Stat Treat. 2020;3:482-8.

2. Helfenstein S, Riesterer O, Meier UR, Papachristofilou A, Kasenda B, Pless M, et al. 3-weekly or weekly cisplatin concurrently with radiotherapy for patients with squamous cell carcinoma of the head and neck - a multicentre, retrospective analysis. Radiat Oncol. 2019;14(1):32. https://doi. org/10.1186/s13014-019-1235-y.

3. Tsan D-L, Lin C-Y, Kang C-J, Huang S-F, Fan K-H, Liao C-T, et al. The comparison between weekly and three-weekly cisplatin delivered concurrently with radiotherapy for patients with postoperative high-risk squamous cell carcinoma of the oral cavity. Radiat Oncol. 2012;7(1):215. https://doi.org/10.1186/1748-717X-7-215.

4. Szturz P, Wouters K, Kiyota N, Tahara M, Prabhash K, Noronha V, et al. Altered fractionation radiotherapy combined with concurrent low-dose or high-dose cisplatin in head and neck cancer: a systematic review of literature and meta-analysis. Oral Oncol. 2018;76:52-60. https://doi.org/10.1 016/j.oraloncology.2017.11.025.

5. Singh GK, Capoor MR, Nair D, Bhowmik KT. Spectrum of fungal infection in head and neck cancer patients on chemoradiotherapy. J Egypt Natl Canc Inst. 2017;29(1):33-7. https://doi.org/10.1016/j.jnci.2017.01.006.

6. Patil VM, Noronha V, Joshi A, Agarwal J, Ghosh-Laskar S, Budrukkar A, et al. A randomized phase 3 trial comparing nimotuzumab plus cisplatin chemoradiotherapy versus cisplatin chemoradiotherapy alone in locally advanced head and neck cancer. Cancer. 2019;125(18):3184-97. https://doi. org/10.1002/cncr.32179.

7. National Cancer Institute. Common terminology criteria for adverse events (CTCAE) v4.03. Bethesda: National Institutes of Health, National Cancer Institute; 2010. https://www.eortc.be/services/doc/ctc/CTCAE_4.03_2010-
06-14_QuickReference_5x7.pdf. Published May 28, 2009. Accessed 12 Feb 2021

8. Mallick S, Benson R, Rath GK. Radiation induced oral mucositis: a review of current literature on prevention and management. Eur Arch Otorhinolaryngol. 2016;273(9):2285-93. https://doi.org/10.1007/s00405-015-3 694-6.

9. Bernier J, Domenge C, Ozsahin M, Matuszewska K, Lefèbvre JL, Greiner RH, et al. Postoperative irradiation with or without concomitant chemotherapy for locally advanced head and neck cancer. N Engl J Med. 2004;350(19): 1945-52. https://doi.org/10.1056/NEJMoa032641.

10. Cooper JS, Pajak TF, Forastiere AA, Jacobs J, Campbell BH, Saxman SB, et al. Postoperative concurrent radiotherapy and chemotherapy for high-risk squamous-cell carcinoma of the head and neck. N Engl J Med. 2004;350(19): 1937-44. https://doi.org/10.1056/NEJMoa032646.

11. Noronha V, Joshi A, Patil VM, Agarwal J, Ghosh-Laskar S, Budrukkar A, et al. Once-a-week versus once-every-3-weeks cisplatin chemoradiation for locally advanced head and neck cancer: a phase iii randomized noninferiority trial. J Clin Oncol. 2018;36(11):1064-72. https://doi.org/10.1200/JCO.2017.74.9457.

12. Biswal BM. Current trends in the management of oral mucositis related to cancer treatment. Malays J Med Sci. 2008;15(3):4-13.

13. Naidu MU, Ramana GV, Rani PU, Mohan IK, Suman A, Roy P. Chemotherapyinduced and/or radiation therapy-induced oral mucositis--complicating the treatment of cancer. Neoplasia. 2004;6(5):423-31. https://doi.org/10.1593/ neo.04169.

14. Suresh A, Varma PP, Sinha S, Deepika S, Raman R, Srinivasan M, et al. Riskscoring system for predicting mucositis in patients of head and neck cancer receiving concurrent chemoradiotherapy [rssm-hn]. J Can Res Ther. 2010; 6(4):448-51. https://doi.org/10.4103/0973-1482.77100.

15. Trotti A, Bellm LA, Epstein JB, Frame D, Fuchs HJ, Gwede CK, et al. Mucositis incidence, severity and associated outcomes in patients with head and neck cancer receiving radiotherapy with or without chemotherapy: a systematic literature review. Radiother Oncol. 2003;66(3):253-62. https://doi.org/10.101 6/S0167-8140(02)00404-8.

16. Wood BG, Wanamaker JR, Eliachar I, Strome M, Van Kirk MA. A phase III randomized trial comparing concurrent chemotherapy and radiotherapy with radiotherapy alone in resectable stage III and IV squamous cell head and neck cancer: preliminary results. Head Neck. 1997;19(7):567-75.

17. Lalla RV, Sonis ST, Peterson DE. Management of oral mucositis in patients who have cancer. Dent Clin N Am. 2008;52(1):61-77. https://doi.org/10.101 6/j.cden.2007.10.002

\section{Publisher's Note}

Springer Nature remains neutral with regard to jurisdictional claims in published maps and institutional affiliations.

\section{Submit your manuscript to a SpringerOpen ${ }^{\circ}$ journal and benefit from:}

- Convenient online submission

- Rigorous peer review

- Open access: articles freely available online

- High visibility within the field

- Retaining the copyright to your article

Submit your next manuscript at $\boldsymbol{\nabla}$ springeropen.com 